\title{
Verification with the utility of an established rapid assessment of brain safety for newly developed vaccines
}

\author{
Gwang-Ho Kim and Sun Shin Yi*
}

\begin{abstract}
In the twenty-first century, high contagious infectious diseases such as SARS (Severe Acute Respiratory Syndrome), MERS (Middle East Respiratory Syndrome), FMD (Foot-and-Mouth Disease) and Al (Avian Influenza) have become very prevalent, causing treat harm to humans and animals in aspect of public health, and economical issues. The critical problem is that newly-reported infectious diseases that humans firstly experience are expected to continue to emerge, and these diseases will be spreading out rapidly. Therefore, rapid and safe supplies of effective vaccines are most pivotal to prevent the rapid prevalent of new infection, but international standards or assessing protocol the safety of urgent vaccines are not established well. In our previous study, since we established a module to assess the brain safety of urgent vaccines, therefore, it is necessary to verify that this established module for assessing brain safety could work effectively in commercially available two vaccines (one killed-and on livevaccines). We compared the results of Evans blue (EB) assay and qPCR analysis by injection of two kinds of vaccines, PBS and Lipopolysaccharide (LPS) under the condition of the module previously reported. We confirmed that the brain safety test module for urgent vaccine we established is very reproducible. Therefore, it is believed that this vaccine safety testing method can be used to validate brain safety when prompt supply of a newly developed vaccines is needed.
\end{abstract}

Keywords: Brain, Infectious disease, Public health, Rapid assessment, Vaccine safety

\section{Introduction}

Recently, a variety of infectious diseases with high rates of spread and mortality have been frequent. In addition, it has been reported that high contagious infectious diseases are spreading through various routes and carriers for recent two decades. It is important to prevent from spreading out a number of infectious diseases earlier in the outbreak of the causing agents because of our rapid transportation population density. Therefore, prompt supplying of vaccines should be secured within a limit time, even at unexpected times and places. The speed of vaccine development is also a very critical issue. Thus, safety of the vaccines also should be assured. Nevertheless, it is difficult to obtain sufficient time to perform a complete safety evaluation for a rapid supply of urgent vaccines. It is very unfortunate that we have already

\footnotetext{
* Correspondence: admiral96@sch.ac.kr

Department of Biomedical Laboratory Science, Soonchunhyang University, Asan 31538, Republic of Korea
}

mentioned in the previous report that there is not global standard for the safety assessment of these newly develop urgent vaccines.

In our previous study, we established of minimal positive control conditions to ensure brain safety using experimental mice models during development of urgent vaccines before introducing to markets. We conducted several experiments to evaluate the safety of vaccines, and presented the ideal combination of the experiments: susceptible mice types according to the effective concentration of lipopolysaccharide (LPS) destroying Bloodbrain barrier (BBB), the appropriate LPS exposure time destroying BBB, origin type of LPS (Salmonella enterica), and injection route of LPS. In this study, we tried to evaluate whether the previously established assessment method could be available for evaluating brain safety rapidly with two commercial vaccines easily accessible vaccines (a killed- and a live-vaccine). We confirmed in the present study that the pre-established "vaccine safety 
module for the brain" was highly reproducible in the evaluation of the safety results by the commercially available vaccines.

In this study, we described that this module can be useful in applying a commercially available vaccine to a rapid and effective positive-control for brain safety assessment of an urgent vaccine. This study will be of great help to ensure that vaccines should be urgently and promptly distributed to public with assured minimal safety of brain at an unexpected outbreak of certain infectious diseases in human and veterinary fields.

\section{Materials and methods}

Most of experiments were performed based on our previous study. Among the experiments, we primarily chose some combinations that we'd suggested in the previous reports by Baek et al., [1].

In brief, evidences for the BBB damage by commercially available vaccines (killed porcine genital respiratory syndrome vaccine vaccine and live canine parvovirus vaccine) were based on Evans blue EB permeability into brain parenchyma and mRNA expression of tight junctions (ZO-1 and Occludin) in the brain. Experimental method for this is described in detail below.

\section{Evans blue (EB) assay for brain permeability}

Seven-weeks old ICR mice and LPS sources Salmonella enterica were used. LPS solutions concentration was 1.0 $\mathrm{mg} / 4 \mathrm{ml} / \mathrm{kg}$, and i.p injection was performed. The LPS from S.enterica serotype enteritidis (L6011; Sigma, USA) was dissolved in phosphate buffered saline (PBS; pH 7.4). Two types of commercially available vaccines were used as experimental groups. One is a killed vaccine, a porcine genital respiratory syndrome vaccine (Suishot PRRS, Choongang vaccine, Korea). The other one is a live vaccine, a canine parvovirus vaccine (Zoetis Inc., USA). The concentration of the stock solution of these two vaccines is $0.5 \times 10_{7.0} \mathrm{TCID}_{50} / \mathrm{ml}$. We set these concentrations to assess the effect of these vaccines on the $\mathrm{BBB}$, depending on the concentration. Through the previous studies we confirmed that the effective $\mathrm{BBB}$ destroyed concentration of these vaccines was 2 -fold concentration of the stock solutions. Therefore, the experiment was conducted under three conditions: 2-fold concentration of the stock solution, reference concentration of the stock solution and 10-fold diluted concentration of the stock solution. Including negative control and positive control, the groups were divided 8 groups according to vaccine types and concentrations: group 1, $\mathrm{PBS} / \mathrm{EB}(n=2)$; group2, $\mathrm{LPS} / \mathrm{EB}(\mathrm{n}=2)$; group3, 2-fold concentration of the porcine vaccine/EB (P1, $n=5)$; group4, reference concentration of the porcine vaccine/EB (P2, $n=5)$; group 5, 10-fold diluted concentration of the porcine vaccine/EB (P3, $n=5)$; group6, 2-fold concentration of the canine vaccine/EB $(\mathrm{C} 1, n=5)$; group7, reference concentration of the canine vaccine/EB $(C 2, n=5)$; group 8,10 fold diluted concentration of the canine vaccine/EB (C3, $n=5$ ). We performed the EB assay according to the method of Baek et al. [1], used in previous studies.

For treatment, PBS, LPS and the vaccines were intraperitoneally (i.p) injected into each mouse $(1.0 \mathrm{mg} / 4 \mathrm{~mL} /$ $\mathrm{kg}$ body weight). At $1 \mathrm{~h}$ post-injection (PI) of PBS/LPS/ Vaccines, EB dye $(2 \% \mathrm{w} / \mathrm{v}$ in PBS, $4 \mathrm{~mL} / \mathrm{kg}$, E2129; Sigma) was i.p injected into each mouse. All mice were sacrificed after $3 \mathrm{~h}$ of EB exposure (4h PI). After sacrifice, blood was collected and serum was separated by using centrifugation. The brain was promptly removed after the mouse had been perfused with heparinized PBS $(0.1 \mathrm{mg} / \mathrm{L})$. Serum and homogenized brain hemisphere of each mouse were dissolved in a $50 \%(\mathrm{w} / \mathrm{v})$ trichloroacetic acid in PBS solution to eliminate proteins and then subjected to centrifugation at $5000 \times g$ for 20 min. Supernatant of each sample was diluted 1:3 with absolute ethanol.

A spectrophotometer (Infinite F200; Tecan, Switzerland) was used to determine the EB concentrations in the serum and brain hemisphere samples. The fluorescence intensity was measured at $620 \lambda_{\text {ex }}$ and $680_{\mathrm{em}}$, and the EB concentration of each sample was calculated according to a standard curve that had been ordered to determine the penetration rate for $\mathrm{EB}$ in brain to EB in blood was calculated.

\section{Brain tissue collection and preparation of RNA samples}

Following the EB assay, the safety of the vaccine was assessed by mRNA expression of the molecules forming the tight junction of the brain. Groups and experimental conditions were used same as EB assay. The LPS solution was i.p. injected into all mice and sacrificed at the 4 $\mathrm{h}$ PI time. After sacrifice, the brains were removed from each mouse and were prepared for next steps. RNA was extracted by using Trizol reagent (Ambion; Life Technologies, USA) according to the manufacture's instruction. For RNA extraction, the homogenized brain tissue was incubated in Trizol and chloroform reagent and then subjected to centrifugation. The aqueous phase was removed and incubated with $100 \%$ isopropanol. After centrifugation, the obtained RNA pellet was washed with $75 \%$ ice-cold ethanol and then dissolved in RNase-free water. The obtained RNA extract solution was stored at $-70^{\circ} \mathrm{C}$ after undergoing $60^{\circ} \mathrm{C}$ heat incubation.

\section{Quantification of mRNA expression of ZO-1 and occluding in the brain}

Quantitative real-time polymerase chain reaction (qPCR) was performed with SYBR Green dye by using a Step One Plus Real-time PCR system (Life Technologies). For relative quantitation of gene expression, we used the 
comparative cycle threshold $(\mathrm{Ct})$ method $\left(2^{-\Delta \Delta C t}\right)$. Results were normalized to that of the housekeeping/control gene glyceraldehyde 3-phosphate dehydrogenase (GAPDH). The primer sequences for zonula occludens-1 (ZO-1) and occludin have been used in our previous studies to establish positive control. These primer sequences were obtained from the National Center for Biotechnology Information nucleotide database and are shown in Table 1.

\section{Data analysis}

Data are presented as mean \pm SE or mean \pm SEM values for each experimental group. Differences between means were analyzed by using Student's $t$-test for single comparisons. $P$ values $<0.05$ were considered statistically significant.

\section{Results}

\section{EB assay for brain permeability}

After i.p injection of PBS, LPS $(1.0 \mathrm{mg} / 4 \mathrm{ml} / \mathrm{kg})$ and two vaccines at three concentrations, the permeability of $\mathrm{EB}$ dye in brain parenchyma was measured by the ratio of the amount of EB dye in brain to the amount of EB dye in blood (Fig. 1). In the first injection before EB injection, the result of the group injected PBS was negative control, and the result of the group injected LPS was positive control. When compared to the PBS/EB group, the permeability of EB dye was significantly increased in mice of the group injected with LPS/EB (Figs.1a and b). The EB dye permeability of P1 group (; injected with 2fold concentration of porcine vaccine stock solution) and P2 group (; injected with reference concentration of porcine vaccine stock solution) was increased than PBS/ EB group, but not significantly, and EB dye penetrated through the brain parenchyma less than LPS/EB group (Fig. 1a). However, the permeation rate of EB dye in $\mathrm{C} 1$ group (; injected with 2-fold concentration of canine vaccine stock solution) was not significant but higher than LPS/EB group (Fig. 1b). The 10-fold diluted group of the two vaccines had similar or lower EB dye permeability than the PBS/EB group.

Table 1 Primer Sequences of ZO-1, occludin, and GAPDH

\begin{tabular}{ll}
\hline Target mRNA & Sequences \\
\hline ZO-1 & Forward 5' - ACA GGC CAT TAC GAG CCT CT - 3' \\
& Reverse 5' - GGA GGC TGT GGT TTG GTA GC - 3' \\
Occludin & Forward 5' - CAC ACA GGA CAT GCC TCC AC - 3' \\
& Reverse 5' - GGC TGC CTG AAG TCA TCC AC - 3' \\
GAPDH & Forward 5' - GAC GGC CGC ATC TTC TTG T-3' \\
& Reverse 5' - CAC ACC GAC CTT CAC CAT TाT - 3'
\end{tabular}

Sequences of primers used for quantitative real-time PCR analysis. The sequence of the primers was used with reference to the previous description of Baek et al. [1]. ZO-1, zonula occludens-1; GAPDH, glyceraldehyde 3-phosphate dehydrogenase
Quantification of ZO-1 and occludin mRNA expressions in the brain

The relative ZO-1 and occludin mRNA levels were plotted on the graph after $4 \mathrm{~h}$ after i.p injection with PBS, LPS, and two vaccines at three concentrations (Fig. 2). ZO-1 mRNA levels were significantly higher in the LPS group (positive control) than in the PBS group (negative control). In addition, ZO-1 mRNA levels were significantly increased compared to PBS group when 2-fold concentrations of two vaccine stock solutions were injected. At the 2-fold concentration of the stock solution, ZO-1 mRNA expression was lower in the P1 group (; porcine vaccine) but, higher in the $\mathrm{C} 1$ group (; canine vaccine) than the LPS group, but not significantly. The ZO-1 mRNA expressions were similar or slightly higher in the P2 and C2 groups (; reference concentration of two vaccines stock solution) compared to the PBS group. And the ZO-1 mRNA expression was similar or slightly lower in the P3 and C3 groups (; 10-fold diluted concentration of two vaccines stock solution) compared to the PBS group.

Likewise, occludin mRNA levels were significantly higher in the LPS group (positive control) than in the PBS group (negative control). However, when 2-fold concentrations of two vaccines stock solutions were injected, occludin mRNA expression was increased compared to the PBS group, but not significantly. In addition, the increase was smaller than ZO-1 mRNA level. In the P2, C2, P3 and C3 groups injected with reference concentrations and 10-fold diluted concentrations of the two vaccines, occludin expression was similar or slightly higher than the PBS group.

\section{Discussion}

Humans and live stocks are easily exposed to the risk of infectious diseases caused by various micro-organisms such as bacteria and virus on a daily basis. This has become a very important issue as it affects health in our daily lives [1]. Once a high contagious infectious disease occurs, it can spread to other countries in an instant [4]. Thus, it is not limited to any region or country. Rapid supply of vaccines that are effective in emergency situations is essential to prevent the spread of such infectious diseases [15]. However, in urgent situation, both quick supply of vaccine and safe vaccine are important. All important biological actions of humans and live stocks are regulated and maintained primarily by the central nervous system (CNS), especially the brain $[1,14,16]$. Brain is a complex network of numerous neurons. It is an important organ for regulating body homeostasis, behaviors, memory, and learning [20-22]. This important organ is protected by a structure known as the bloodbrain barrier (BBB) [8]. Neurons in the brain and spinal cord are in directly contact with cerebrospinal fluid 


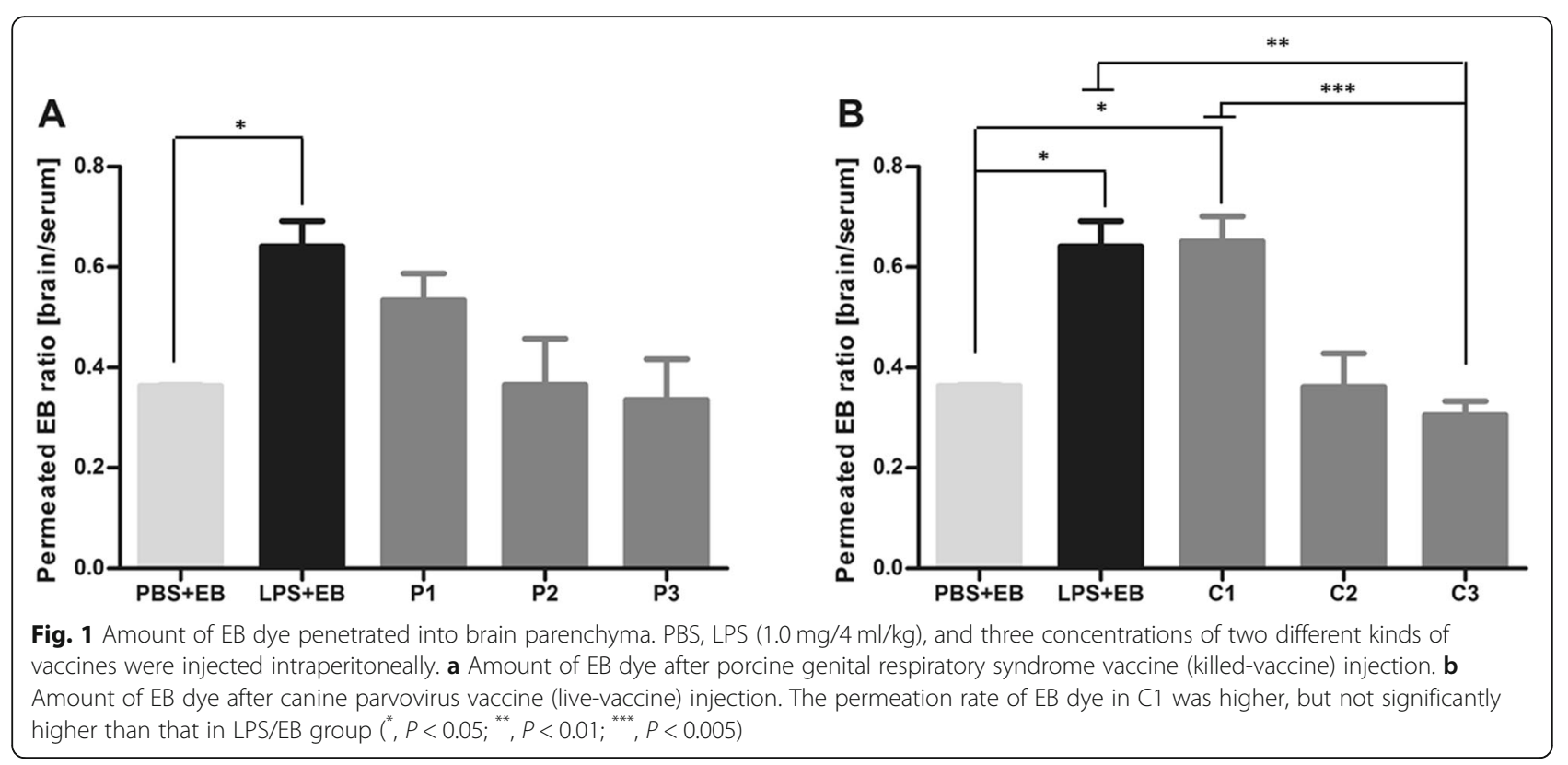

(CSF) [19]. They are supplied with oxygen and nutrients. When blood and CSF circulate, the material can selectively penetrate because the $\mathrm{BBB}$ limits mass transfer into brain parenchyma $[11,18]$. It is believed that the brain can examine components of blood and only let required substances selectively pass [2]. If the BBB is exposed to any toxic substance, chronic high blood pressure, radiation, viruses, or bacteria, it can lead to brain problem $[3,18]$. If a newly developed vaccine or urgent vaccine fails to secure brain safety, brain parenchyma might become damaged and the health of the recipient may not be assured [1]. This can become can be a serious public health hazard and result in serious economic damage to industrial animals. Therefore, the safety assessment module we designed was focused on how to effectively measure effects of various vaccines on the stability of $\mathrm{BBB}$ in laboratory animals [1]. The BBB is formed by a tight junction between endothelial cells of blood vessels distributed throughout the CNS. It contains junctional molecules (JAM), zonula occludens ( $\mathrm{ZO})$, claudin, and occluding [3, 5-7, 9-12]. These structures are key components of tight junction of $\mathrm{BBB}[3,5-7,9,10]$. In our previous study, we have established a condition for positive control that can destroy the BBB structure by damage to the tight junction using LPS mentioned earlier [1]. These conditions include mouse species, age, type, concentration of LPS, injection routes to mice, and exposure time after injection. EB assay, qPCR, and Western blot analysis can be conducted under the condition of positive control to determine whether the $\mathrm{BBB}$ is destroyed $[2,3,9,11,13,17,18]$. In the present study, we confirmed that these established conditions and modules of the positive control that destroyed the BBB could be used to assess brain safety of commercial vaccines [1].

Results of EB assay revealed that EB dye permeability of P1 group was higher than that of the $\mathrm{PBS} / \mathrm{EB}$ group but lower than that of the LPS/EB group. Based on this, we could not conclude that the BBB was damaged in the vaccine concentration of the $\mathrm{P} 1$ group. On the other hand, the permeability of $\mathrm{EB}$ dye in the $\mathrm{C} 1$ group was similar or higher than that in the LPS/EB group, meaning that $\mathrm{BBB}$ was damaged at vaccine concentration of C1 group. The same concentration of vaccine was injected. However, higher permeability of EB dye was seen in the $\mathrm{C} 1$ group. This result may be due to different vaccine types of porcine and canine vaccines. Since the porcine vaccine is a killed-vaccine and the canine vaccine is a live-vaccine, the canine vaccine is more damaging to BBB. This was also confirmed by qPCR results of ZO-1 mRNA levels. As expected, at reference concentration and 10-fold diluted concentration of stock solution of two vaccines, EB dye permeability and relative ZO-1 and occludin mRNA levels were comparable to those of the negative control, meaning no significant damage to the BBB. Occludin mRNA expression was lower than ZO-1 mRNA expression level at 2-fold diluted concentration of the stock solution which was considered to be damaging to $\mathrm{BBB}$ [1]. We have previously examined permeability of EB dye and changes in mRNA and protein expression of $\mathrm{ZO}-1$ and occludin over time after LPS injection. After $4 \mathrm{~h}$ of LPS injection, ZO-1 mRNA levels were dynamic and rapidly increased. The level of occludin mRNA increased after $4 \mathrm{~h}$ of injection, but increased rapidly after $24 \mathrm{~h}$. These results are highly correlated with our previous findings. However, in order 


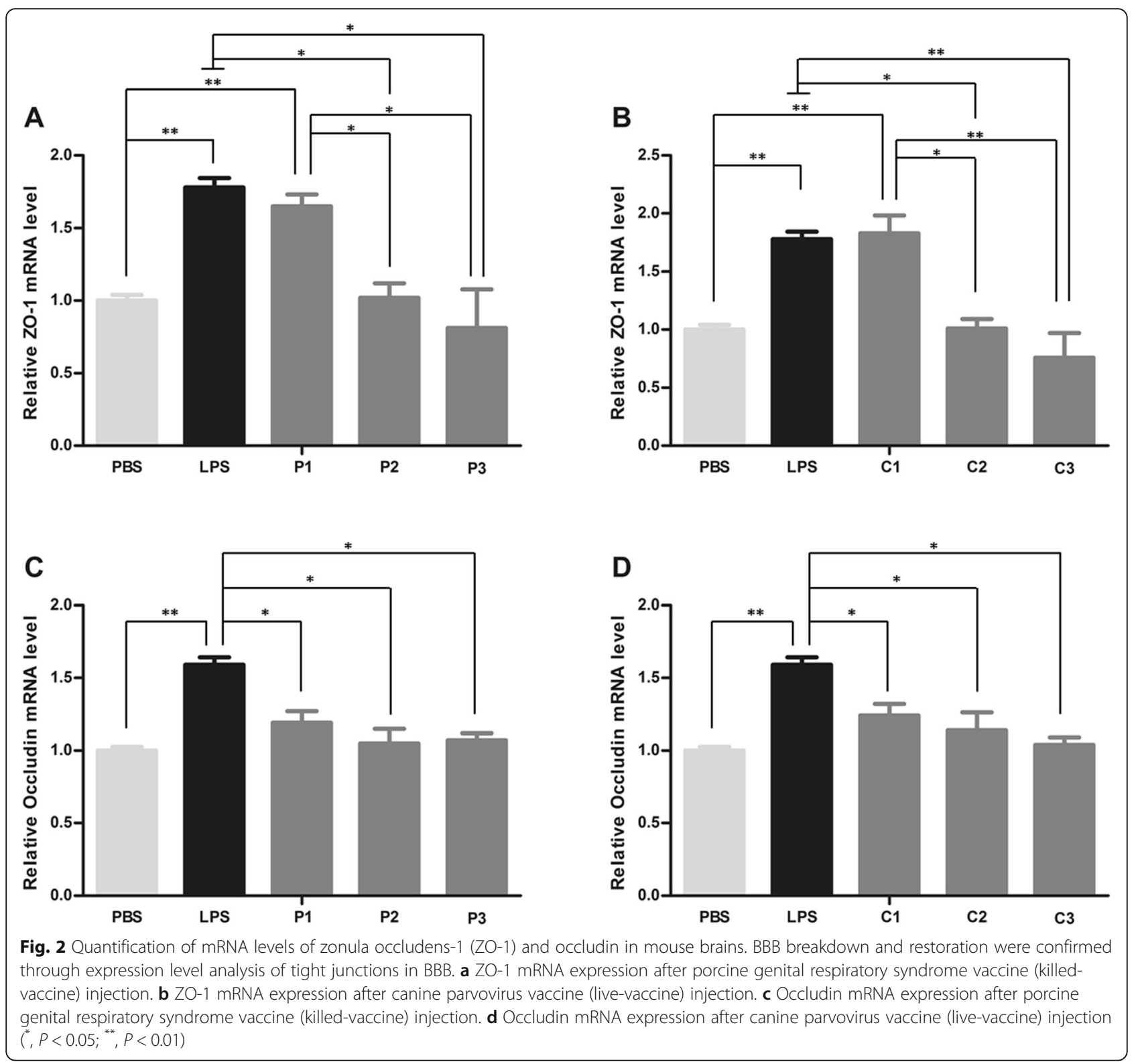

to provide a rapid supply of urgent vaccines, rapid verification is required. Considering this, $4 \mathrm{~h}$ after injection of LPS was established as a positive control condition of the module for evaluating BBB damage. Consistent with results of previous studies, ZO-1 levels, but not occludin levels, were increased rapidly after $4 \mathrm{~h}$ of vaccine injection. Therefore, when a commercially available vaccine is applied to our positive control and rapid safety assay modules, ZO-1 tight junction primer is more suitable for qPCR. In addition, we have purposed three assays of EB assay, qPCR, and western blot as methods to determine damage to BBB in our previous study [1]. However, since this module is aimed at assessing the safety of urgent vaccines, western blot analysis which requires a relatively long time might not be suitable. Therefore, EB assay and
qPCR would be more effective methods for rapid evaluation. It is recommended that we refer to an illustration of our research process on the safety assessment of vaccines (Fig. 3).

\section{Conclusion}

In this study, we confirmed that the safety assessment module for urgent vaccines could be used to evaluate two commercial vaccines. However, in order for this module to be used in practice, it is necessary to apply more types of vaccines to the module. Since there are many kinds of vaccines, they should be repeatedly applied and evaluated using this module. Taken together, the usefulness of the rapid brain safety test module that we constructed previously was verified using vaccines 


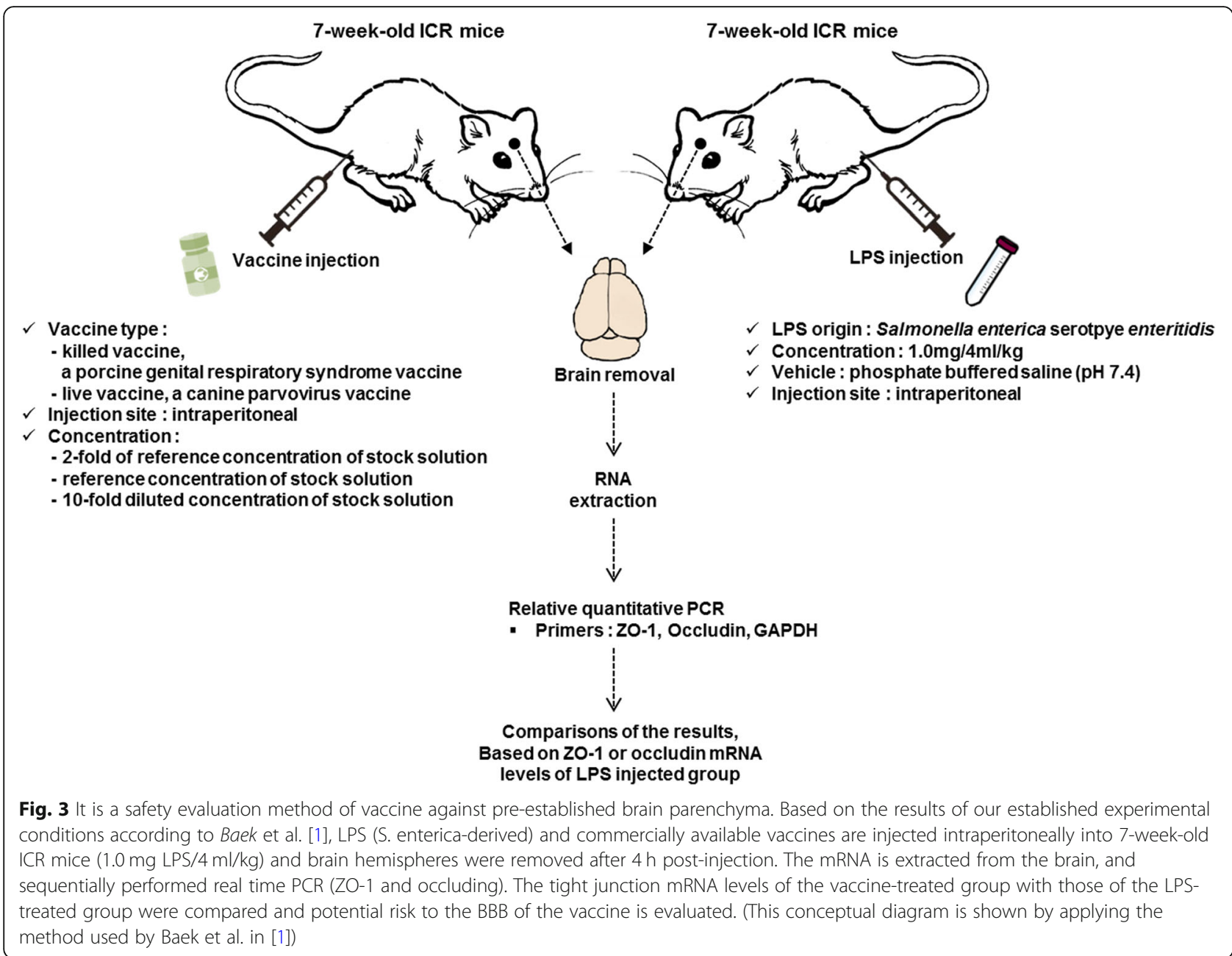

commercially available in the veterinary market in Korea. Our results were highly reproducible. Therefore, this evaluation module can be utilized in the future to assess the safety of various vaccines and adjuvants.

\section{Acknowledgments}

All authors are appreciated the technical supports of Mr. Kyunghyun Lim and Mr. Ji-Kwang Lee at department of biomedical laboratory science, Soonchunhyang University.

\section{Authors' contributions}

GHK; performing experiment, analysis of data and writing manuscript, SSY; designing of the study, analysis and interpretation of data and final approval of the version to be submitted. Both authors read and approved the final manuscript.

Authors' information

There were no more related information about the authors.

\section{Funding}

This work was supported by the National Research Foundation of Korea Grant funded by the Korean Government (NRF-2015R1C1A1A02037060) and Soonchunhyang University Research Fund.
Availability of data and materials

There was some supporting data available for this work. The datasets used and/or analyzed in this study are available from the corresponding author on reasonable request.

\section{Competing interests}

The authors declare that they have no competing interests.

Received: 4 October 2019 Accepted: 14 November 2019

Published online: 29 November 2019

\section{References}

1. Baek H, Kim KH, Park MY, Kim K, Ko B, Seo HS, Kim BS, Hahn TW, Yi SS. Establishment of minimal positive-control conditions to ensure brain safety during rapid development of emergency vaccines. J Vet Sci. 2017;18(S1): 371-9.

2. Ballabh P, Braun A, Nedergaard M. The blood-brain barrier: an overview: structure, regulation, and clinical implications. Neurobiol Dis. 2004;16(1): $1-13$

3. Banks WA, Gray AM, Erickson MA, Salameh TS, Damodarasamy M, Sheibani N, Meabon JS, Wing EE, Morofuji Y, Cook DG, Reed MJ. Lipopolysaccharideinduced blood-brain barrier disruption: roles of cyclooxygenase, oxidative stress, neuroinflammation, and elements of the neurovascular unit. J Neuroinflammation. 2015;12:223.

4. Barreto ML, Teixeira MG, Carmo EH. Infectious diseases epidemiology. J Epidemiol Community Health. 2006;60(3):192-5. 
5. Cereijido M, Contreras RG, Shoshani L, Flores-Benitez D, Larre I. Tight junction and polarity interaction in the transporting epithelial phenotype. Biochim Biophys Acta. 2008;1778(3):770-93.

6. Cummins PM. Occludin: one protein, many forms. Mol Cell Biol. 2012;32(2): 242-50.

7. Edelblum KL, Turner JR. The tight junction in inflammatory disease: communication breakdown. Curr Opin Pharmacol. 2009;9(6):715-20.

8. Elia L, Quintavalle M, Zhang J, Contu R, Cossu L, Latronico MV, Peterson KL, Indolfi C, Catalucci D, Chen J, Courtneidge SA, Condorelli G. The knockout of miR-143 and -145 alters smooth muscle cell maintenance and vascular homeostasis in mice: correlates with human disease. Cell Death Differ. 2009. 16(12):1590-8

9. Fanning AS, Jameson BJ, Jesaitis LA, Anderson JM. The tight junction protein ZO-1 establishes a link between the transmembrane protein occludin and the actin cytoskeleton. J Biol Chem. 1998:273(45):29745-53.

10. Fu BM. Experimental methods and transport models for drug delivery across the blood-brain barrier. Curr Pharm Biotechnol. 2012:13(7):1346-59.

11. Gunzel D, Yu AS. Claudins and the modulation of tight junction permeability. Physiol Rev. 2013:93(2):525-69.

12. Huber JD, Egleton RD, Davis TP. Molecular physiology and pathophysiology of tight junctions in the blood-brain barrier. Trends Neurosci. 2001;24(12): $719-25$

13. Jangula A, Murphy EJ. Lipopolysaccharide-induced blood brain barrier permeability is enhanced by alpha-synuclein expression. Neurosci Lett. 2013; 551:23-7.

14. Kim JH, Yu JE, Chang BJ, Nahm SS. Neonatal influenza virus infection affects myelination in influenza-recovered mouse brain. J Vet Sci. 2018;19(6):750-8.

15. Kitler ME, Gavinio P, Lavanchy D. Influenza and the work of the World Health Organization. Vaccine. 2002;20(Suppl 2):S5-14.

16. Kondo T, Yoshida S, Nagai H, Takeshita A, Mino M, Morioka H, Nakajima T, Kusakabe KT, Okada T. Transient forebrain ischemia induces impairment in cognitive performance prior to extensive neuronal cell death in Mongolian gerbil (Meriones unguiculatus). J Vet Sci. 2018;19(4):505-11.

17. Qin LH, Huang W, Mo XA, Chen YL, Wu XH. LPS Induces Occludin Dysregulation in Cerebral Microvascular Endothelial Cells via MAPK Signaling and Augmenting MMP-2 Levels. Oxid Med Cell Longev. 2015; 2015:120641.

18. Stamatovic SM, Keep RF, Andjelkovic AV. Brain endothelial cell-cell junctions: how to "open" the blood brain barrier. Curr Neuropharmacol. 2008;6(3):17992.

19. Vigh B, Manzano e Silva MJ, Frank CL, Vincze C, Czirok SJ, Szabo A, Lukats A, Szel A. The system of cerebrospinal fluid-contacting neurons. Its supposed role in the nonsynaptic signal transmission of the brain. Histol Histopathol 2004; 19(2): 607-628.

20. Waterson MJ, Horvath TL. Neuronal regulation of energy homeostasis: beyond the hypothalamus and feeding. Cell Metab. 2015;22(6):962-70.

21. Yi SS. Time-dependent changes of calbindin D-28K and parvalbumin immunoreactivity in the hippocampus of rats with streptozotocin-induced type 1 diabetes. J Vet Sci. 2013;14(4):373-80.

22. Yi SS, Hwang IK, Kim YN, Kim IY, Pak SI, Lee IS, Seong JK, Yoon YS. Enhanced expressions of arginine vasopressin (Avp) in the hypothalamic paraventricular and supraoptic nuclei of type 2 diabetic rats. Neurochem Res. 2008;33(5):833-41.

\section{Publisher's Note}

Springer Nature remains neutral with regard to jurisdictional claims in published maps and institutional affiliations.

Ready to submit your research? Choose BMC and benefit from:
- fast, convenient online submission
- thorough peer review by experienced researchers in your field
- rapid publication on acceptance
- support for research data, including large and complex data types
- gold Open Access which fosters wider collaboration and increased citations
- maximum visibility for your research: over 100M website views per year
At BMC, research is always in progress.
Learn more biomedcentral.com/submissions

